\title{
RAINIER GRUTMAN,Des langues qui résonnent
}

\section{Roberto Ferraroni}

\section{OpenEdition \\ Journals}

\section{Édition électronique}

URL : https://journals.openedition.org/studifrancesi/45599

DOI : 10.4000/studifrancesi.45599

ISSN : 2421-5856

\section{Éditeur}

Rosenberg \& Sellier

\section{Édition imprimée}

Date de publication : 1 août 2021

Pagination : 419-420

ISSN : 0039-2944

\section{Référence électronique}

Roberto Ferraroni, « RAINIER Grutman, Des langues qui résonnent », Studi Francesi [En ligne], 194 (LXV | II) | 2021, mis en ligne le 01 septembre 2021, consulté le 08 décembre 2022. URL : http://

journals.openedition.org/studifrancesi/45599; DOI : https://doi.org/10.4000/studifrancesi.45599

Ce document a été généré automatiquement le 15 octobre 2022.

\section{(c) (†)}

Creative Commons - Attribution - Pas d'Utilisation Commerciale - Pas de Modification 4.0 International - CC BY-NC-ND 4.0

https://creativecommons.org/licenses/by-nc-nd/4.0/ 


\title{
RAINIER GRUTMAN,Des langues qui résonnent
}

\author{
Roberto Ferraroni
}

\section{RÉFÉRENCE}

RAINIER GRUTMAN,Des langues qui résonnent, Paris, Classiques Garnier, 2019, 359 pp.

1 L'étude que Rainier Grutman présente est un parcours qui se déroule dans les méandres de la littérature québécoise, en priorité du XIX ${ }^{\mathrm{e}}$ siècle. Paru pour la première fois en 1997, en 2019 Grutman a décidé d'en publier une nouvelle édition, revue, corrigée, actualisée, enrichie par de nouvelles réflexions et observations. En particulier, l'étude focalise son attention sur l'hétérolinguisme très répandu dans la littérature du Québec. Il propose ce nouveau terme pour indiquer «les manières dont les langues se font écho à l'intérieur d'un texte» (p. 59), pour mettre en évidence les modalités d'intégration de langues, souvent très différentes, dans une œuvre littéraire. Le thème du bilinguisme ou même du plurilinguisme a toujours été très cher au Québec, et au Canada en général, comme Grutman en informe les lecteurs dans la longue et très intéressante introduction à son étude. En effet le Québec, sans aucun doute à cause de sa position géographique et de son histoire, se trouve dans une situation très particulière parce que deux langues officielles peuvent être utilisées dans la vie quotidienne et au Parlement: le français et l'anglais; en outre, ses lois doivent obligatoirement être écrites dans les deux langues officielles. Cette réalité peut pousser le lecteur à penser que les Québécois sont en majorité bilingue; au contraire, Grutman s'appuie sur des données précises pour démontrer que la plupart des Québécois ne maîtrise à fond qu'une seule langue, qui dépend de la région où ils vivent. En tous cas, il souligne également comment les deux langues s'influencent et se contaminent réciproquement. Naturellement, la même chose se produit dans le domaine littéraire.

2 Dans le premier chapitre, l'auteur cherche à brosser le tableau de la communauté linguistique et des comportements sociolinguistiques, en abordant deux notions qui 
peuvent se ressembler mais qui en réalité sont différentes: diglossie, «plus opératoire en l'occurrence que le mot bilinguisme, très courant mais imprécis et lourdement connoté» (p. 44). Il s'agit d'une analyse claire et minutieuse qui trace nettement la différence entre ces deux notions: alors qu'avec le mot bilinguisme on indique la caractéristique d'une personne qui connait et communique couramment en deux langues, qui parfois peuvent s'interpénétrer quand on parle, la diglossie est rigoureusement plus rigide, parce que dans ce cas chaque langue est consacrée à une fonction bien définie et il n'y a aucune possibilité que l'une d'entre elles débouche sur un «terrain» qui ne lui appartient pas. Après cette explication, Grutman propose certains exemples de bilinguisme et de diglossie littéraires en abordant même le thème de l'hétérolinguisme dans les œuvres des auteurs canadiens. Il met en évidence comment les littératures anglaise et française ont exercé leur influence sur la production littéraire des colonies d'outre-atlantique. Il donne une définition très claire d'hétérolinguisme, en soulignant les différences avec des termes comme par exemple plurilinguisme ou multilinguisme, qui ont des limites comparables, parce qu'«ils traduisent une volonté de mettre de l'ordre (homogène) dans ce qui est perçu comme du désordre (hétérogène)» (p. 64). Au contraire, «l'hétérolinguisme signifie aussi la (con)fusion, le mélange, l'hybridité» (p. 64). Le dernier paragraphe est consacré à un excursus dans les genres littéraires québécois touchés par l'hétérolinguisme, en particulier les romans, les contes et la nouvelle, alors que la poésie et le théâtre ne sont concernés que marginalement par ce phénomène.

3 À partir du chapitre suivant, Grutman s'éloigne un peu de l'aspect purement théorique et aborde une analyse très attentive de certaines œuvres québécoises à l'appui de ses thèses. En particulier, le deuxième et le troisième chapitre se focalisent sur le bilinguisme et sont tout à fait complémentaires. En effet le deuxième est consacré au bilinguisme mis en exergue, supporté par des exemples très détaillés, tirés surtout du roman L'Influence d'un livre de Philippe-Ignace-François Aubert de Gaspé, qui a été inspiré par Walter Scott dans la rédaction de son œuvre: les nombreux termes et les phrases en anglais que l'auteur introduit dans son texte sont les témoins de cette influence. Par contre, le troisième chapitre se focalise au début encore sur le bilinguisme, mais en soulignant qu'il s'agit d'un terme qui doit être dépassé quand on parle de la littérature québécoise. C'est donc à ce moment-là, en analysant le roman de Patrice Lacombe La Terre paternelle, que Grutman introduit un concept nouveau: la tétraglossie qui constitue un aspect fondamental des textes québécois. En effet on ne trouve pas seulement le français et l'anglais dans ces textes, mais au contraire il y a souvent du latin (dans les cérémonies religieuses) et, surtout, des langues vernaculaires, des dialectes, les langues du peuple. On peut donc dire que «le Québec tel que le construisent les œuvres du XIX ${ }^{e}$ siècle est structuré par l'imbrication de quatre codes linguistiques: 1) le langage vernaculaire du peuple québécois; 2) le langage véhiculaire de la nouvelle mère patrie (l'Angleterre); 3) le langage référentiaire de l'ancienne mère patrie (la France) et 4) le langage mythique du rite catholique» (p. 147).

Le chapitre suivant semble se détacher un peu du thème de l'hétérolinguisme (même s'il est toujours présent à l'arrière-plan) pour permettre à Grutman de s'occuper d'une hiérarchie qui existe au sein des différentes œuvres littéraires: cette hiérarchie est étroitement liée au langage choisi par les auteurs, un choix qui est souvent fait pour exalter l'histoire du peuple canadien. Par exemple dans les Légendes canadiennes de l'abbé Casgrain on utilise fréquemment «un langage à la fois trop naïf et trop fleuri pour pouvoir être le calque d'un idiome réel sous-jacent. C'est un miroir poétique dont 
le tain ne reflète pas une image fidèle» (p. 165). Également, dans Les anciens Canadiens d'Aubert Gaspé, l'auteur introduit souvent des expressions latines pour élever le niveau du texte.

5 Alors que le dernier chapitre se concentre sur l'analyse du conte, en étudiant plusieurs exemples de ce genre dans la littérature québécoise, l'avant dernier se révèle une sorte de voyage à travers l'Amérique du nord pour en découvrir les langues et les particularités qui marquent les différentes régions. C'est un voyage très intéressant et étroitement lié à l'hétérolinguisme parce que Grutman montre des exemples de textes où les dialectes entrent en contact et se mêlent avec la langue officielle. Il démontre donc que chaque zone du pays est caractérisée par ses propres langues vernaculaires; et cet aspect se reflète dans la production littéraire de ses auteurs.

Dans la conclusion, Grutman fait le point et résume ses propos, en soulignant encore la multiplicité des langues qui font vivre le monde québécois. Dans cette étude fort intéressante et bien conduite, l'auteur s'appuie sur une écriture très simple et claire, même si souvent il se sert, justement, des termes techniques qu'il explique toujours de façon très détaillée, aussi à travers de bons exemples à l'appui de ses thèses. Le choix de rééditer ce texte s'est révélé un bon choix; Grutman a eu la possibilité d'approfondir certains aspects au vu d'une nouvelle sensibilité réservée au langues vernaculaires qui peuplent l'univers linguistique nord-américain. Ajoutons aussi que Grutman réussit à bien faire émerger les multiples facettes de la langue et de la culture québécoises, et canadiennes en général, ce qui ne saurait manquer d'éveiller la curiosité des lecteurs. 